\title{
Modelocked quantum dot vertical external cavity surface emitting laser
}

\author{
M. Hoffmann • Y. Barbarin • D.J.H.C. Maas • \\ M. Golling • I.L. Krestnikov • S.S. Mikhrin • \\ A.R. Kovsh · T. Südmeyer · U. Keller
}

Received: 14 October 2008 / Published online: 25 October 2008

(C) The Author(s) 2008. This article is published with open access at Springerlink.com

\begin{abstract}
We report the first successful modelocking of a vertical external cavity surface emitting laser (VECSEL) with a quantum dot (QD) gain region. The VECSEL has a total of 35 QD-layers with an emission wavelength of about $1060 \mathrm{~nm}$. In SESAM modelocked operation, we obtain an average output power of $27.4 \mathrm{~mW}$ with 18 -ps pulses at a repetition rate of $2.57 \mathrm{GHz}$. This QD-VECSEL is used asgrown on a $450 \mu \mathrm{m}$ thick substrate, which limits the average output power.
\end{abstract}

PACS $42.55 . P x \cdot 42.60 . F c$

\section{Introduction and motivation}

Optically pumped vertical external cavity surface emitting lasers (VECSELs) offer high output power, excellent beam quality and good efficiency [1]. They produced average output powers as high as $30 \mathrm{~W}$ with an $M^{2}$ value of 3 [2] or $20 \mathrm{~W}$ with an $M^{2}$ value below 1.1 [3]. The output power can be scaled up simply by increasing the mode area on the gain region and the pump power if the heat flow through the layer structure is nearly one-dimensional [4]. Moreover, semiconductor bandgap engineering can be used to obtain a large variation of emission wavelengths that are not covered

M. Hoffmann ( $\varangle)$ - Y. Barbarin · D.J.H.C. Maas · M. Golling · T. Südmeyer $\cdot$ U. Keller

Department of Physics, ETH Zurich, 8093 Zurich, Switzerland e-mail: mh@phys.ethz.ch

Fax: +41-44-6331059

I.L. Krestnikov · S.S. Mikhrin · A.R. Kovsh Innolume GmbH, Konrad-Adenauer-Allee 11, 44263 Dortmund, Germany by established solid state lasers. In addition, semiconductor lasers are ideally suited for mass production because they are based on a wafer-scale technology with a high level of integration.

In combination with a semiconductor saturable absorber mirror (SESAM) [5], VECSELs have been passively modelocked, and average output powers as high as $2.1 \mathrm{~W}$ [6], pulse repetition rates as high as $50 \mathrm{GHz}$ [7] and pulses as short as 260 fs [8] were achieved. We recently introduced a new concept of ultrafast semiconductor lasers, where we integrated the saturable absorber and the active region into a single semiconductor based device. We refer to this new class of modelocked VECSELs as modelocked integrated external-cavity surface emitting lasers (MIXSEL) [9]. One key requirement was the development of quantum dot saturable absorbers that support the integration with the same laser mode size in the absorber and the gain. The MIXSEL platform has a high potential for applications in optical communication, optical clocking of multi-core microprocessors and compact supercontinuum generation for bio-medical applications. However, so far we always used quantum wells (QWs) for the gain section, and the pulse duration has been limited to the picosecond regime. Our motivation for using quantum dot (QD) gain structures within a MIXSEL or a modelocked VECSEL is the larger inhomogeneous broadening. Self-assembled InAs/GaAs QDs produce an inhomogenously broadened gain [10], which is wider compared to QWs and is less susceptible to temperature changes [11]. Previously, we have demonstrated with diode-pumped solidstate lasers that inhomogenous gain broadening relaxes the requirements on the saturable absorber and supports shorter pulse durations $[12,13]$. With inhomogeneous broadening, the lasing bandwidth is increased at continuous wave (cw) operation because spectral hole burning enables many different axial modes to lase at the same time. Therefore, 
the saturable absorber only needs to provide sufficient selfamplitude modulation to phase-lock these modes without having to transfer the full energy from one axial mode to all other adjacent modes. We believe that the inhomogeneous broadening of QD-gain will enable sub-picosecond pulse generation of modelocked VECSELs and MIXSELs with fast quantum dot saturable absorbers $[14,15]$ in the near future.

VECSELs based on QD-gain regions have recently been reported in cw-operation at an emission wavelength of $1034 \mathrm{~nm} \mathrm{[16]} \mathrm{and} 1210 \mathrm{~nm}$ [17], where output powers of $1.4 \mathrm{~W}$ and $300 \mathrm{~mW}$, respectively, were achieved using diamond heat spreaders.

Here, we report on the first passively modelocked QDgain VECSEL. We employed a standard quantum well SESAM (QW-SESAM) and obtained self-starting stable pulses of 18-ps duration with an average output power of $27.4 \mathrm{~mW}$ at a pulse repetition rate of $2.57 \mathrm{GHz}$. The average output power of $27.4 \mathrm{~mW}$ is currently limited by the reduced thermal conductivity of the $450 \mu \mathrm{m}$ thick GaAs wafer, onto which the VECSEL structure has been grown.

\section{Laser cavity, QD-VECSEL gain structure and SESAM}

We use a typical V-shaped laser cavity, which is shown in Fig. 1 [18]. The QD-VECSEL is pumped at $808 \mathrm{~nm}$ at an angle of 45 degrees. A QW-SESAM and a curved output coupling mirror constitute the end-mirrors of the laser cavity. The operation wavelength can be tuned by changing the

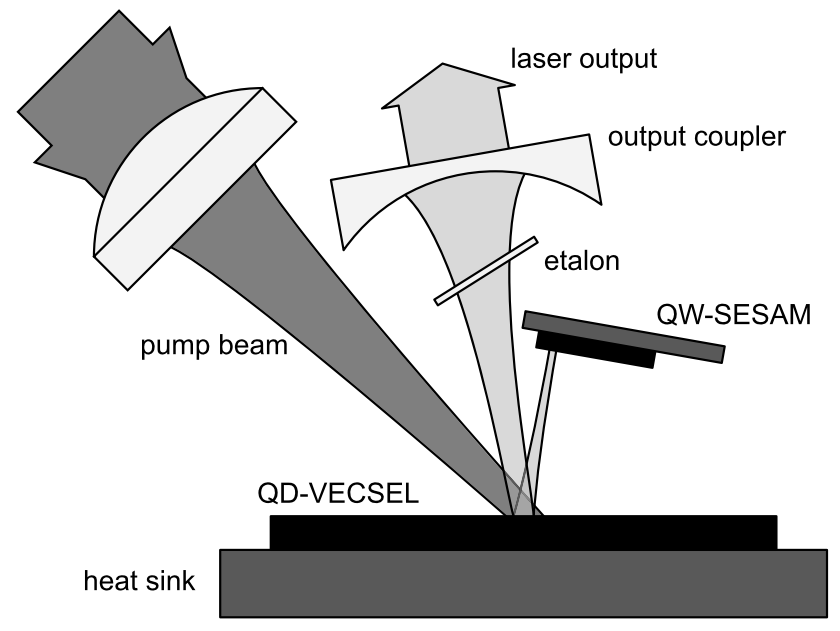

Fig. 1 Schematic of the laser setup. A V-shaped laser cavity is used with a quantum well SESAM (QW-SESAM) and the output coupler as the cavity end-mirrors. Inside the cavity we used a quantum dot gain VECSEL (QD-VECSEL) structure and a $20 \mu \mathrm{m}$ thick etalon to tune the central wavelength of the laser. The 808-nm pump light is focused onto the QD-VECSEL structure under an angle of 45 degrees angle of a $20-\mu \mathrm{m}$ thick etalon. In this configuration the pulse passes the active region twice per cavity roundtrip.

The QD-VECSEL gain structure was grown by Innolume $\mathrm{GmbH}$ by molecular beam epitaxy (MBE) on a GaAs substrate of $450-\mu \mathrm{m}$ thickness. The structure consists of a distributed Bragg reflector (DBR) centered around 1060-nm wavelength and an active $\mathrm{QD}$ region. The $\mathrm{DBR}$ is made of 29 pairs of quarter wavelength layers of GaAs and $\mathrm{Al}_{0.9} \mathrm{Ga}_{0.1} \mathrm{As}$, which results in a high reflectivity at the laser wavelength (calculated 99.99\%). The active region consists of five stacks of seven QD-layers embedded in GaAs, whereas the GaAs serves as absorbing medium for the pump radiation. In each stack, the seven QD-layers are separated from each other by $10 \mathrm{~nm}$ using GaAs spacers, and the five stacks are placed in five different antinodes of the standing wave pattern of the electrical field of the laser.

The QD stacks were formed by cyclic deposition of InAs (1/4 monolayer thickness) and GaAs (1/3 monolayer thickness) followed by short growth interruptions. The substrate temperature was kept as low as 510 to $520^{\circ} \mathrm{C}$, monitored by an infrared pyrometer. No dislocations have been detected in the grown structure.

The SESAM used for modelocking the QD-VECSEL contains a QW saturable absorber in a standard anti-resonant design [19]. It was grown by MBE in the FIRST cleanroom facility at ETH Zurich and consists of a 30-pair AlAs/GaAs DBR centered at a wavelength of $1062 \mathrm{~nm}$ and a single InGaAs QW saturable absorber. The saturable absorption parameters of the SESAM were characterized with a high-precision nonlinear reflectivity setup [20]. At 1064-nm center wavelength, we determined a saturation fluence of $34 \mu \mathrm{J} / \mathrm{cm}^{2}$, a modulation depth of $0.6 \%$ and nonsaturable losses of $0.2 \%$.

The length of the laser cavity was $58.5 \mathrm{~mm}$, which corresponds to a repetition rate of $2.6 \mathrm{GHz}$. The full folding angle of the cavity was 10 degrees. The dielectric output coupling mirror had $0.28 \%$ transmission at the laser wavelength and a radius of curvature of $60 \mathrm{~mm}$. The round pump spot on the gain chip had $90 \mu \mathrm{m}$ radius and the pump beam had an $M^{2}$ value of 12 . The cavity geometry was chosen such that the laser mode radius on the SESAM was about $50 \mu \mathrm{m}$.

For our experiment, the QD-VECSEL structure was directly soldered onto a heat sink, and no further steps were taken towards improved thermal management [4]. During modelocked laser operation, the heat sink temperature was set to $-20^{\circ} \mathrm{C}$.

\section{Experimental results and discussion}

Fundamental modelocking with an average output power of $27.4 \mathrm{~mW}$ was achieved for a pump power of $1.56 \mathrm{~W}$ (Fig. 2). Pulses of 18-ps duration were measured with a second harmonic generation autocorrelator. We measured the optical 

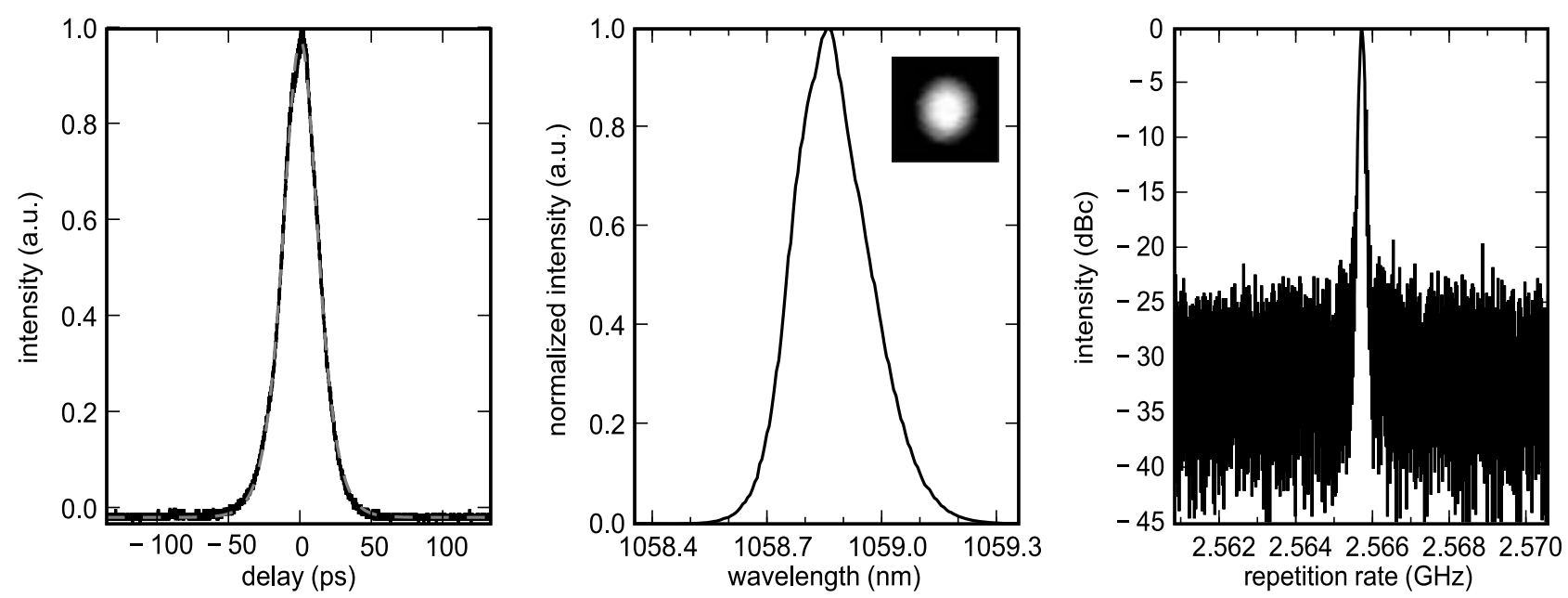

Fig. 2 The autocorrelation trace (black) and its fit (gray dashed), the emission spectrum with the beam profile as inset and the microwave spectrum are plotted for a pump power of $1.56 \mathrm{~W}$ on the QD-VECSEL. The deconvoluted pulse has 18 ps FWHM duration; the width of the optical spectrum is $0.22 \mathrm{~nm}$, measured with a resolution bandwidth of $0.1 \mathrm{~nm}$. The microwave spectrum shows a clear peak at $2.57 \mathrm{GHz}$, the spectral span was $10 \mathrm{MHz}$ with a resolution bandwidth of $100 \mathrm{kHz}$ spectrum with an optical spectrum analyzer that had a resolution bandwidth of $0.1 \mathrm{~nm}$ and obtained an FWHM width of $0.22 \mathrm{~nm}$. A simple deconvolution would result in an FWHM width of $0.2 \mathrm{~nm}$ and a time bandwidth product of 1 (i.e. about 3 times the transform limit of a $\operatorname{sech}^{2}$ pulse). The microwave spectrum is measured over a span of $10 \mathrm{MHz}$ with a resolution bandwidth of $100 \mathrm{kHz}$. We see a clear peak at $2.57 \mathrm{GHz}$, and additional measurements also clearly show the peaks for the higher harmonics of this frequency. In Fig. 2, also the beam profile of the laser beam is shown as an inset within the emission spectrum of the laser.

For pump powers higher than $1.56 \mathrm{~W}$, a thermal rollover of the average power occurred. This roll-over is caused by the low thermal conductivity of the VECSEL structure. We did not optimize thermal management because this experiment was a proof-of-principle for modelocking a QDVECSEL. Thus the $450 \mu \mathrm{m}$ thick GaAs substrate has not been removed, and the average power is limited by thermal heat sinking [4]. The advantage of this structure is that almost no additional processing is required before the device can be used in the laser cavity.

We numerically simulated the temperature increase within our structure using a finite-element method for a pump spot of $90 \mu \mathrm{m}$ and a pump power of $1.5 \mathrm{~W}$. We obtained a difference in temperature between the active region and the heat sink of more than $100 \mathrm{~K}$. Lasing experiments with cw-output of the VECSEL in a straight cavity setup also confirmed that the output power level is limited by the absolute temperature in the active region of the VECSEL. These numerical simulations furthermore show that the temperature difference between the heat sink and the active region can be reduced to $25 \mathrm{~K}$ for equally high pump powers if the $450 \mu \mathrm{m}$ thick GaAs substrate is removed from the VECSEL and the grown structure is soldered directly onto the heat sink. We expect that such a QD-VECSEL will be power-scalable to several watts in analogy to QWVECSELs. We would expect that a faster QD-SESAM and better dispersion management [21, 22] will ultimately result in shorter pulse durations.

\section{Conclusion and outlook}

We have demonstrated the first modelocked QD-VECSEL. Pulses of 18-ps duration with a repetition rate of $2.57 \mathrm{GHz}$ were generated at a laser wavelength of $1059 \mathrm{~nm}$. We could achieve an average output power of $27.4 \mathrm{~mW}$. The average power performance of the laser can be further improved by optimizing the thermal properties of the VECSEL structure by substrate removal. The ongoing development of faster SESAMs using quantum dots and improved cavity dispersion management should allow the generation of femtosecond pulses in the near future.

Acknowledgements This work was supported in part by the European Community's Seventh Framework Programme FAST-DOT under grant agreement 224338, the Intel Corporation through a university sponsored research agreement, and ETH Zurich with the FIRST cleanroom facility. We thank Oleg Okhotnikov from the Tampere University of Technology and Edik Rafailov from the University of Dundee for fruitful discussions.

Open Access This article is distributed under the terms of the Creative Commons Attribution Noncommercial License which permits any noncommercial use, distribution, and reproduction in any medium, provided the original author(s) and source are credited. 


\section{References}

1. M. Kuznetsov, F. Hakimi, R. Sprague, A. Mooradian, High-power ( $>0.5-\mathrm{W} \mathrm{CW}$ ) diode-pumped vertical-external-cavity surfaceemitting semiconductor lasers with circular TEM $_{00}$ beams. IEEE Photonics Technol. Lett. 9, 1063-1065 (1997)

2. J. Chilla, S. Butterworth, A. Zeitschel, J. Charles, A. Caprara, M. Reed, L. Spinelli, High power optically pumped semiconductor lasers, in Photonics West 2004, Solid State Lasers XIII: Technology and Devices, in Proc. SPIE, vol. 5332, pp. 143-150 (2004)

3. B. Rudin, D.J.H.C. Maas, A.-R. Bellancourt, M. Hoffmann, E. Gini, T. Südmeyer, U. Keller, Highly efficient optically pumped vertical emitting semiconductor laser with more than $20-\mathrm{W}$ average output power in a fundamental transverse mode. Opt. Lett. (2008, to be published)

4. R. Häring, R. Paschotta, A. Aschwanden, E. Gini, F. MorierGenoud, U. Keller, High-power passively mode-locked semiconductor lasers. IEEE J. Quantum Electron. 38, 1268-1275 (2002)

5. U. Keller, K.J. Weingarten, F.X. Kärtner, D. Kopf, B. Braun, I.D. Jung, R. Fluck, C. Hönninger, N. Matuschek, J. Aus der Au, Semiconductor saturable absorber mirrors (SESAMs) for femtosecond to nanosecond pulse generation in solid-state lasers. IEEE J. Sel. Top. Quantum Electron. 2, 435-453 (1996)

6. A. Aschwanden, D. Lorenser, H.J. Unold, R. Paschotta, E. Gini, U. Keller, 2.1-W picosecond passively mode-locked externalcavity semiconductor laser. Opt. Lett. 30, 272-274 (2005)

7. D. Lorenser, D.J.H.C. Maas, H.J. Unold, A.-R. Bellancourt, B. Rudin, E. Gini, D. Ebling, U. Keller, 50-GHz passively modelocked surface-emitting semiconductor laser with $100 \mathrm{~mW}$ average output power. IEEE J. Quantum Electron. 42, 838-847 (2006)

8. K. Wilcox, Opt. Lett. (2008, submitted)

9. D.J.H.C. Maas, A.-R. Bellancourt, B. Rudin, M. Golling, H.J. Unold, T. Südmeyer, U. Keller, Vertical integration of ultrafast semiconductor lasers. Appl. Phys. B 88, 493-497 (2007)

10. L. Harris, D.J. Mowbray, M.S. Skolnick, M. Hopkinson, G. Hill, Emission spectra and mode structure of InAs/GaAs self-organized quantum dot lasers. Appl. Phys. Lett. 73, 969-971 (1998)

11. M. Grundmann, O. Stier, S. Bognar, C. Ribbat, F. Heinrichsdorff, D. Bimberg, Optical properties of self-organized quantum dots: Modeling and experiments. Phys. Status Solidi A Appl. Res. 178, $255-262(2000)$
12. B. Braun, K.J. Weingarten, F.X. Kärtner, U. Keller, Continuouswave mode-locked solid-state lasers with enhanced spatial holeburning, Part I: Experiments. Appl. Phys. B 61, 429-437 (1995)

13. F.X. Kärtner, B. Braun, U. Keller, Continuous-wave-mode-locked solid-state lasers with enhanced spatial hole-burning, Part II: Theory. Appl. Phys. B 61, 569-579 (1995)

14. D.J.H.C. Maas, A.-R. Bellancourt, M. Hoffmann, B. Rudin, M. Golling, T. Südmeyer, U. Keller, Recombination dynamics in quantum dot semiconductor saturable absorber mirrors (QDSESAMs), in Conference on Lasers and Electro-Optics (CLEO), San Jose, California, 2008, p. talk CFU4

15. E.U. Rafailov, S.J. White, A.A. Lagatsky, A. Miller, W. Sibbett, D.A. Livshits, A.E. Zhukov, V.M. Ustinov, Fast quantum-dot saturable absorber for passive mode-locking of solid-state lasers. IEEE Photonics Technol. Lett. 16, 2439-2441 (2004)

16. T.D. Germann, A. Strittmatter, J. Pohl, U.W. Pohl, D. Bimberg, J. Rautiainen, M. Guina, O.G. Okhotnikov, High-power semiconductor disk laser based on InAs/GaAs submonolayer quantum dots. Appl. Phys. Lett. 92, 101123 (2008)

17. T.D. Germann, A. Strittmatter, J. Pohl, U.W. Pohl, D. Bimberg, J. Rautiainen, M. Guina, O.G. Okhotnikov, Temperature-stable operation of a quantum dot semiconductor disk laser. Appl. Phys. Lett. 93, 3 (2008)

18. U. Keller, A.C. Tropper, Passively modelocked surface-emitting semiconductor lasers. Phys. Rep. 429, 67-120 (2006)

19. G.J. Spühler, K.J. Weingarten, R. Grange, L. Krainer, M. Haiml, V. Liverini, M. Golling, S. Schon, U. Keller, Semiconductor saturable absorber mirror structures with low saturation fluence. Appl. Phys. B 81, 27-32 (2005)

20. M. Haiml, R. Grange, U. Keller, Optical characterization of semiconductor saturable absorbers. Appl. Phys. B 79, 331-339 (2004)

21. R. Paschotta, R. Häring, U. Keller, A. Garnache, S. Hoogland, A.C. Tropper, Soliton-like pulse-shaping mechanism in passively mode-locked surface-emitting semiconductor lasers. Appl. Phys. B 75, 445-451 (2002)

22. E.J. Saarinen, R. Herda, O.G. Okhotnikov, Dynamics of pulse formation in mode-locked semiconductor disk lasers. J. Opt. Soc. Am. B Opt. Phys. 24, 2784-2790 (2007) 